\title{
Removal of an extraluminal gastric gastrointestinal stromal tumor: the role of submucosal tunneling endoscopic resection
}
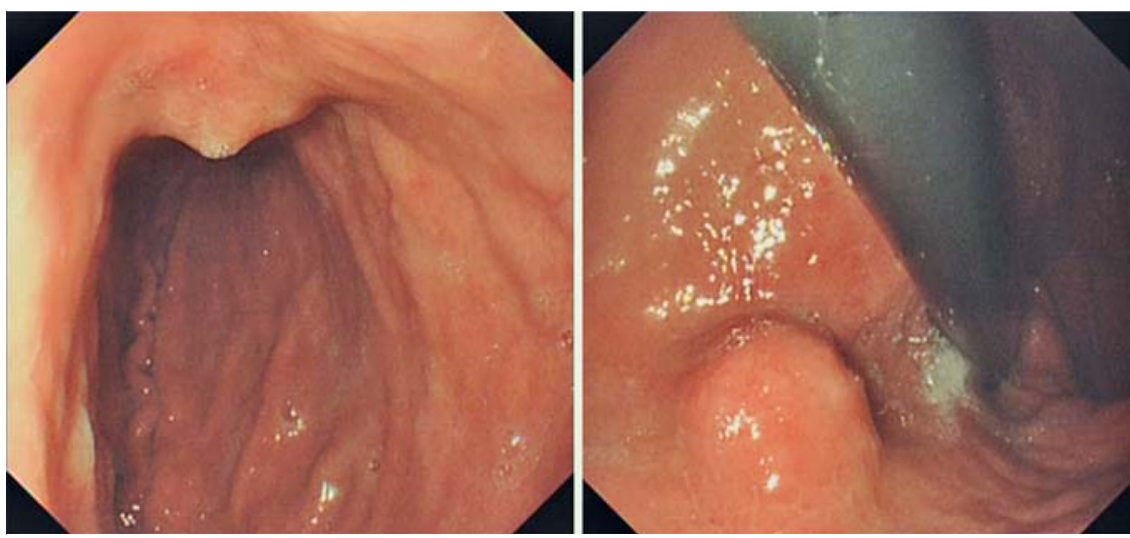

- Fig. 1 Endoscopic images showing a protrusive lesion in the lesser curvature of the stomach body.
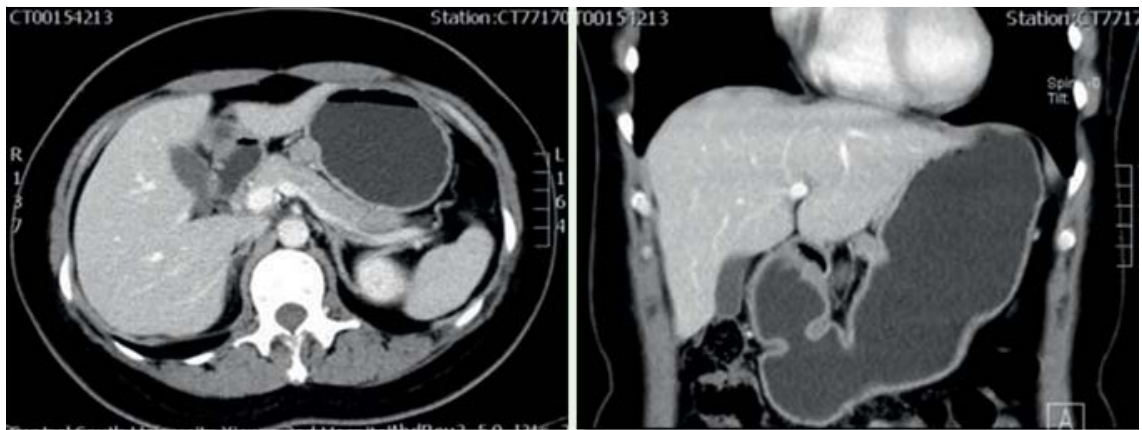

- Fig. 3 Computed tomography (CT) scan showing extraluminal growth of the lesion.

A 47-year-old woman presented with a 10 -year history of epigastric discomfort. She had had a thyroid adenoma treated surgically 2 years previously. Esophagogastroduodenoscopy (EGD) revealed a protrusive lesion at the lesser curvature of the gastric corpus ( $\triangleright$ Fig. 1). Endoscopic ultrasonography (EUS) revealed that the tumor was originating from the serosal layer and was exhibiting extraluminal growth (> Fig.2). Computed tomography (CT) scanning revealed that the lesion was located at the gastric corpus and was protruding into the abdominal cavity ( $\triangleright$ Fig. 3 ; $>$ Video 1 ).

Submucosal tunneling endoscopic resection (STER) was performed ( $\mathbf{F i g . 4}$; - Video 2). After a longitudinal mucosal incision had been made, a submucosal

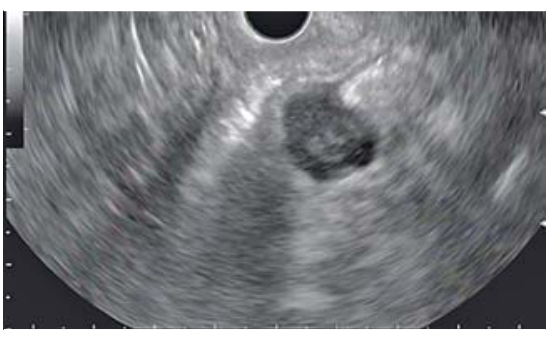

Fig. 2 Endoscopic ultrasonography (EUS) image of the lesion.

tunnel was created, which allowed the lesion to be visualized. The tumor was carefully dissected off, following which, we could see the omentum. The mucosal entry was then closed. The STER procedure was completed uneventfully within 100 minutes. The resected tumor, which measured $2.0 \times 2.0 \times 1.5 \mathrm{~cm}$, was a lowrisk gastrointestinal stromal tumor (GIST) on histopathology ( $>$ Fig. 5).

Surgical or endoscopic removal is recommended for symptomatic or large $(\geq 2 \mathrm{~cm})$ gastric GISTs [1]. Endoscopic resection is a minimally invasive method for GISTs, and current methods include endoscopic submucosal dissection (ESD), endoscopic submucosal excavation, and endoscopic full-thickness resection (EFR) [2]. EFR is feasible for extralum-

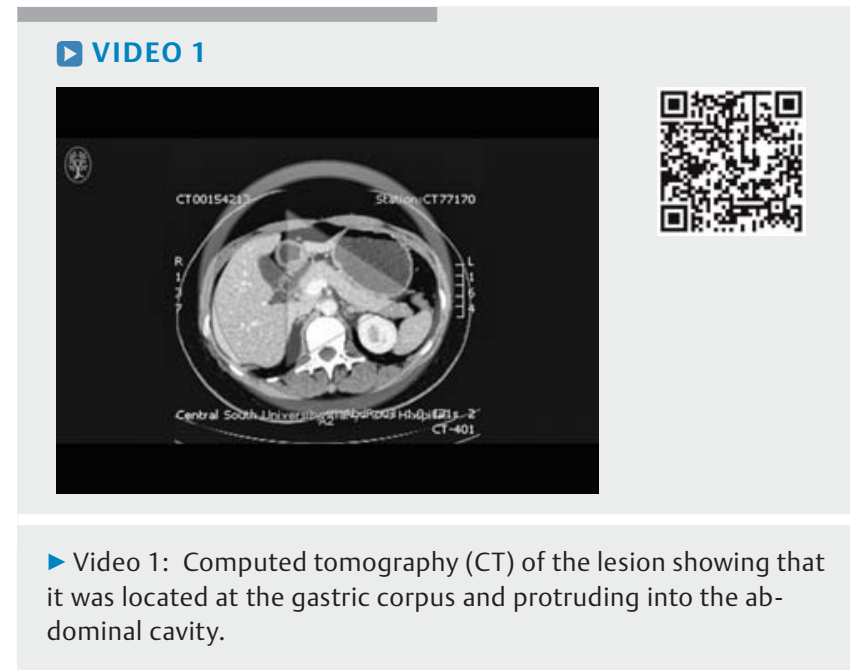




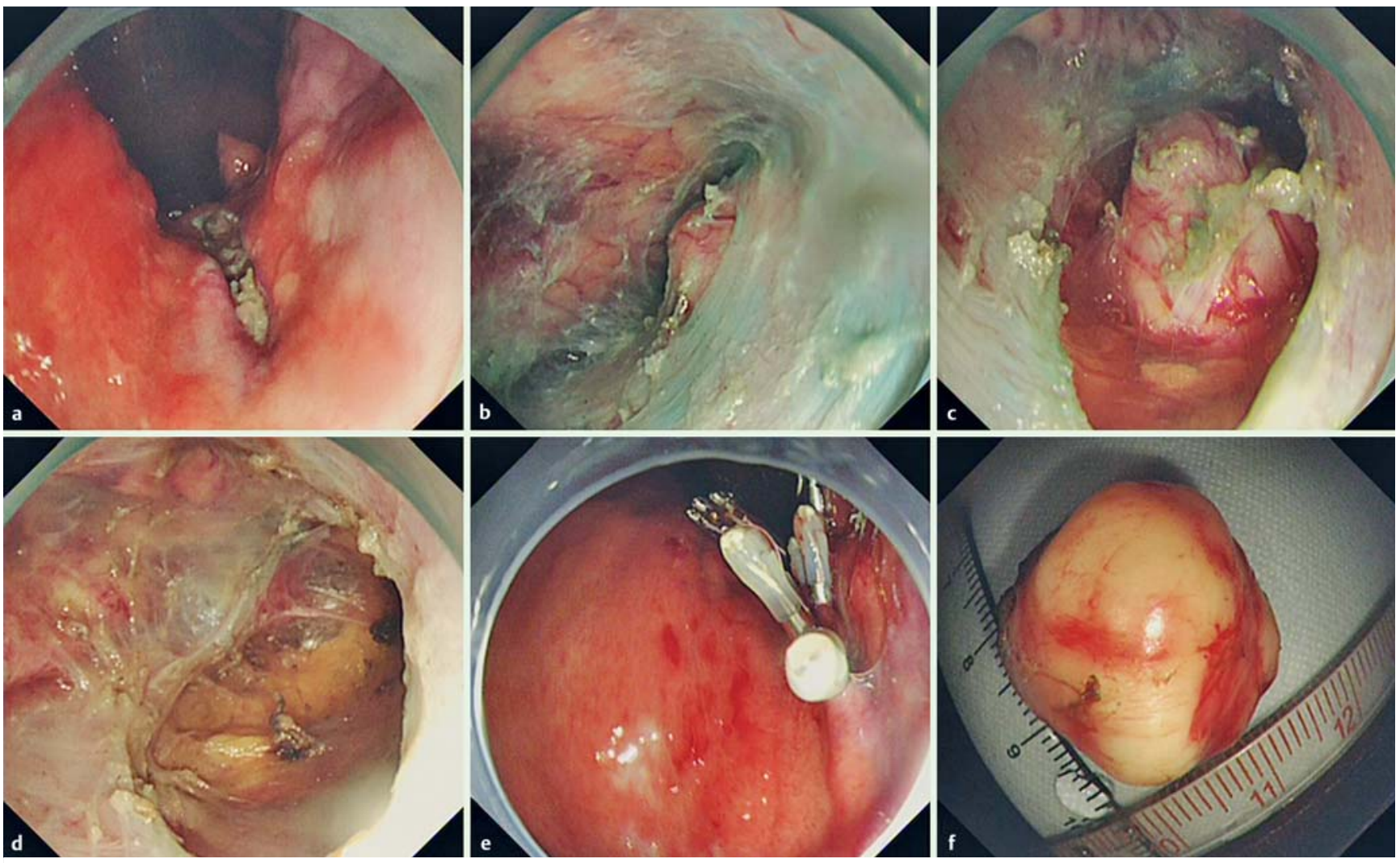

- Fig. 4 The submucosal tunneling endoscopic resection (STER) procedure. a The tunnel entry is created; $\mathbf{b}$ the tumor is identified in the submucosal tunnel; $\boldsymbol{c}$ the tumor in closer view; $\mathbf{d}$ the wound surface after removal of the tumor (note: the omentum is visible); $\mathbf{e}$ the tunnel entry is closed with several clips. $\mathbf{f}$ Macroscopic appearance of the resected tumor.

inal gastric GISTs; however, complications such as perforation, fistula formation, and peritoneal infection may ensue, and closure of the defect is technically difficult [3]. STER can maintain the mucosal integrity and decreases the difficulty of closure of the mucosal defect, which theoretically reduces the risks of the above complications. It has been demonstrated to be safe and effective for treating gastric GIST, although all of the reported cases were intraluminal GISTs [4] [5].

In the present case, we successfully removed an extraluminal gastric GIST using the STER technique. As far as we know, this is the first reported case that has used STER for an extraluminal gastric GIST.

Endoscopy_UCTN_Code_TTT_1AO_2AG

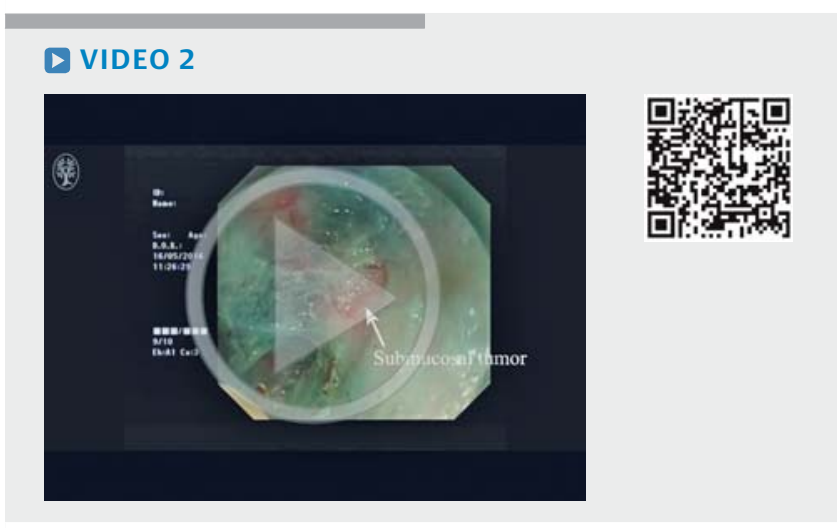

- Video 2: The submucosal tunneling endoscopic resection (STER) procedure. A submucosal injection was made about $5 \mathrm{~cm}$ proximal to the tumor and, after a longitudinal mucosal incision had been made, a submucosal tunnel was created by endoscopic submucosal dissection (ESD). Submucosal injection at the tumor site was used to locate the tumor, which was visualized in the tunnel. The tumor was carefully dissected off, following which, the omentum could be seen. Finally the mucosal entry was closed with several clips. 


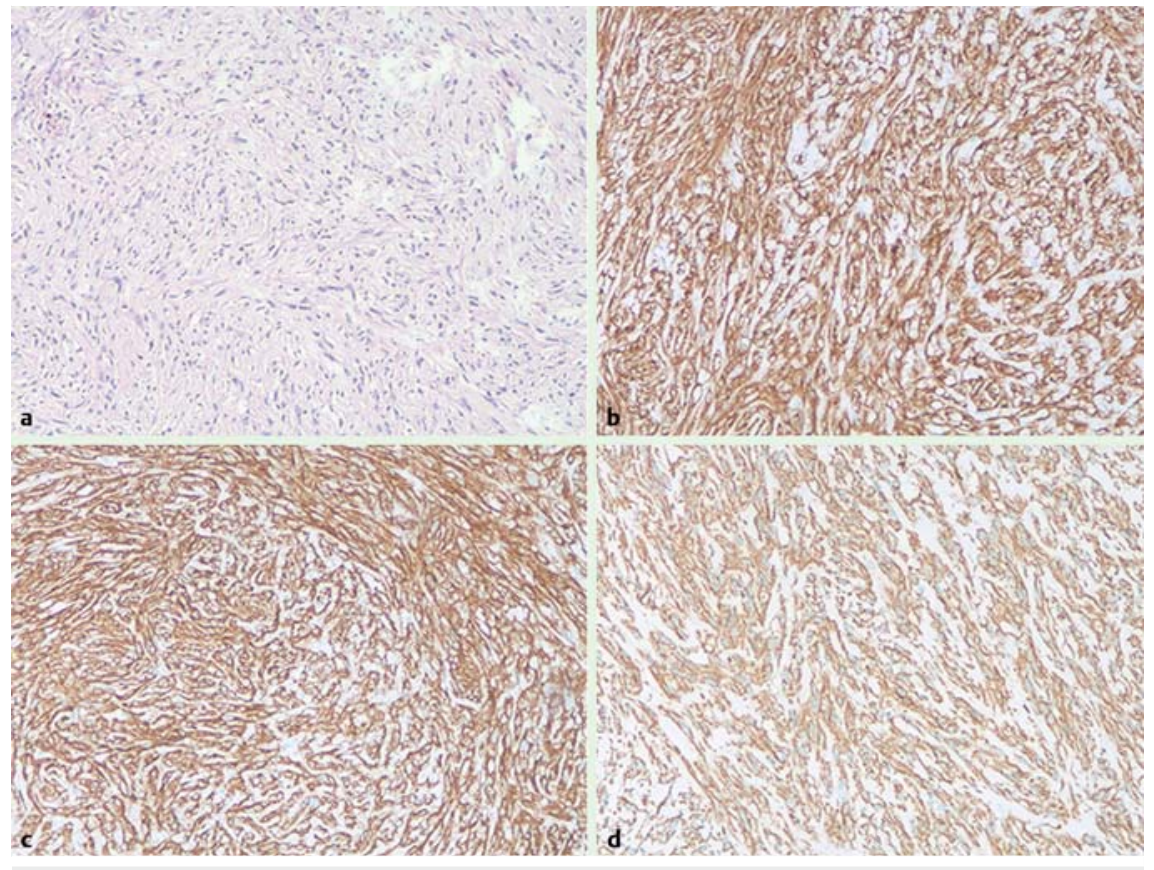

- Fig. 5 Histopathological views of the resected specimen (magnification $\times 100$ ) stained with: a hematoxylin and eosin (H\&E); b CD117; c Dog-1; and d Vim. The findings were consistent with a gastrointestinal stromal tumor (GIST).

The Authors

Hejun Zhou, Yuyong Tan, Chunlian Wang, Jingbo Yang, Yuqian Zhou, Deliang Liu Department of Gastroenterology, The Second Xiangya Hospital of Central South University, Hunan, China

\section{References}

[1] Nishida T, Kawai N, Yamaguchi S et al. Submucosal tumors: comprehensive guide for the diagnosis and therapy of gastrointestinal submucosal tumors. Dig Endosc 2013; 25: 479-489

[2] Ko W], Cho JY. Current techniques for treating gastrointestinal stromal tumors in the upper gastrointestinal tract. Clin Endosc 2016; 49: $226-228$

[3] Mori H, Kobara H, Nishiyama N et al. Review of pure endoscopic full-thickness resection of the upper gastrointestinal tract. Gut Liver 2015; 9: $590-600$

[4] Lu J, Zheng M, Jiao T et al. Transcardiac tunneling technique for endoscopic submucosal dissection of gastric fundus tumors arising from the muscularis propria. Endoscopy 2014; 46: 888-892

[5] Li QL, Chen WF, Zhang C et al. Clinical impact of submucosal tunneling endoscopic resection for the treatment of gastric submucosal tumors originating from the muscularis propria layer (with video). Surg Endosc 2015; 29: 3640 - 3646

\section{Bibliography}

DOI http://dx.doi.org/10.1055/s-0042-119047 Endoscopy 2017; 49: E11-E13

(c) Georg Thieme Verlag KG

Stuttgart · New York

ISSN 0013-726X
Department of Gastroenterology,

The Second Xiangya Hospital of Central South University, NO. 139 Middle Renmin Road, Changsha, Hunan, 410011, China, Fax: +86-0731-85533525,

liudeliang@medmail.com.cn 